\title{
Automated Color Logo Recognition System based on Shape and Color Features
}

\author{
Souvik Ghosh \\ School of Education Technology \\ Jadavpur University \\ Kolkata 700032, India
}

\author{
Ranjan Parekh \\ School of Education Technology \\ Jadavpur University \\ Kolkata 700032, India
}

\begin{abstract}
This paper proposes an automated system for rotation and scale invariant color logo recognition. Colored logo images are recognized using one shape feature namely Moments Invariant and a color feature namely Color Moments. Shape of the logo is modeled using the first two central normalized Hu's invariant moments while color is modeled using the mean, standard deviation, skewness and kurtosis calculated from the color channels. Each image is scaled and rotated by arbitrary amounts before being submitted to the recognition engine. Classification is done using Manhattan and Euclidean distances. Experimental verification is obtained using a data set of 900 images divided into 75 classes. The proposed approach is highly scalable and robust providing accuracy results comparable to the state of the art.
\end{abstract}

\section{General Terms}

Image Processing, Pattern Recognition, Color and Shape features.

\section{Keywords}

Logo Recognition, Moments Invariant, Color Moments, Rotation and Scale Invariance.

\section{INTRODUCTION}

A logo is a graphic mark or symbol commonly used by enterprises and organizations to promote public recognition. Logos may be either purely graphical (only symbol), purely textual (only name of the organization) or textual-graphical (combination of both). Logos are associated with the collective values and brands of organizations and hence their designs are protected by various IPR protocols. This makes a logo unique to a particular organization and provides a visual cue for recognizing and identifying it. Currently, the main applications of $\log$ o recognition systems are in various security and detection agencies where they can be used to track or identify organizations. The challenges in designing a logo recognition system include building a reliable data model to represent arbitrary logo shapes and finding ways of comparing the models with accuracy in real time. Other challenges include handling rotation and scaled variations of the original image. This paper proposes an automated system for rotation and scale invariant color logo recognition based on various shape and color features. The organization of the paper is as follows: section 2 provides an overview of related work, section 3 provides an outline on the proposed approach with discussions on overview, feature extraction and classification schemes, section 4 provides details of the dataset and experimentation results obtained and section 5 provides overall conclusion and future scope for research.

\section{RELATED WORKS}

Many methodologies have been proposed for logo recognition in extant literature. One of the earliest works [1] used negative shape features for Logo Recognition. Negative shape features represent an object that consists of several components enclosed in a simple geometric structure based on its interior with the components considered as holes. Comparison of various local shape descriptors have been presented in [2]. Scale Invariant Feature Transform (SIFT) has been used to detect the interest regions and approximate nearest neighbor is used for efficient matching [3]. In [4] the authors used Fourier Transform and information entropy for E-goods Logo Recognition with Correlation ratio threshold and entropy difference ratio threshold for matching. Various methods have been tested for their effectiveness in Logo Recognition [5] such as Log-Polar Transform (LPT), Fourier-Mellin Transform (FMT) and Gradient Location-Orientation Histogram. In [6] the authors have used Canny Edge Detector to extract object contours for recognition. Other techniques that have been used for logo recognition include Angular Radial Transform [7], radial Tchebichef moments, Zernike Moments, Legendre Moments [8].

\section{PROPOSED APPROACH}

\subsection{Moment Invariants}

For an image the moment of a pixel $P(x, y)$ is defined as the product of the pixel value and its coordinate distances i.e. $m=x \cdot y \cdot P(x, y)$. The moment of an entire image is the summation of moments of all the pixels. The moment of order $(p, q)$ of an image $I(x, y)$ is given by

$$
m_{p q}=\sum_{x} \sum_{y}\left[x^{p} y^{q} I(x, y)\right]
$$

Based on the values of $p, q$ the following moments are defined

$$
\begin{aligned}
m_{00} & =\sum_{x} \sum_{y}[I(x, y)] \\
m_{10} & =\sum_{x} \sum_{y}[x \cdot I(x, y)] \\
m_{01} & =\sum_{x} \sum_{y}[y \cdot I(x, y)] \\
m_{11} & =\sum_{x} \sum_{y}[x \cdot y \cdot I(x, y)] \\
m_{20} & =\sum_{x} \sum_{y}\left[x^{2} \cdot I(x, y)\right] \\
m_{02} & =\sum_{x} \sum_{y}\left[y^{2} \cdot I(x, y)\right]
\end{aligned}
$$

$\mathrm{M}-\mathrm{K} \mathrm{Hu}$ [9] proposed seven moment features that are invariant to rotation. The first two of them are given by

$$
\begin{gathered}
\varphi_{1}=m_{20}+m_{02} \\
\varphi_{2}=\left(m_{20}-m_{02}\right)^{2}+\left(2 m_{11}\right)^{2}
\end{gathered}
$$


To make the moments invariant to translation, the image is shifted such that its centroid coincides with the origin of the coordinate system. The centroid of image in terms of moments is given by:

$$
\begin{aligned}
& x_{c}=m_{10} / m_{00} \\
& y_{c}=m_{01} / m_{00}
\end{aligned}
$$

The central moments are defined as follows:

$$
\mu_{p q}=\sum_{x} \sum_{y}\left[\left(x-x_{c}\right)^{p}\left(y-y_{c}\right)^{q} I(x, y)\right]
$$

To compute Hu moments using central moments the $m$ terms in (3) are replaced by $\mu$ terms.

To make the moments invariant to scaling, the moments are normalized by dividing by a power of $]_{00}$. The normalized central moments are defined as follows:

$$
\begin{aligned}
& \delta_{p q}=\frac{\mu_{p q}}{\left(\mu_{00}\right)^{\omega}} \\
& \omega=1+\frac{p+q}{2}
\end{aligned}
$$

The normalized central moments are defined by substituting the $m$ terms in equation (3) by $\delta$ terms. The first and second central normalized invariant moments of an image $I$ are therefore defined as:

$$
\begin{gathered}
M_{1}(I)=\delta_{20}+\delta_{02} \\
M_{2}(I)=\left(\delta_{20}-\delta_{02}\right)^{2}+\left(2 \delta_{11}\right)^{2}
\end{gathered}
$$

\subsection{Color Moments}

Color Moments are measures that represent the color distribution in an image in a scale and rotation invariant manner. For an RGB image four color moments are computed for each channel leading to a total of 12 color moments. These are explained below:

Mean: The first color moment interpreted as the average value of an image channel and given by:

$$
A_{i}=\frac{1}{N} \sum_{j=1}^{N} P_{i, j}
$$

Here $N$ is the number of pixels in the image and $P_{i, j}$ is the value of the $j$-th pixel of the $i$-th color channel.

Standard Deviation: The second color moment computed by taking the square root of variance of the color distribution.

$$
S_{i}=\sqrt{\frac{1}{N} \sum_{j=1}^{N}\left(P_{i, j}-A_{i}\right)^{2}}
$$

Skewness: The third color moment which measures how asymmetric the color distribution is and gives information about the shape of the color distribution.

$$
W_{i}=\sqrt[3]{\frac{1}{N} \sum_{j=1}^{N}\left(P_{i, j}-A_{i}\right)^{3}}
$$

Kurtosis: The fourth color moment which in addition to shape of color distribution provides information about how tall or flat the distribution is compared to the normal distribution.

$$
K_{i}=\sqrt[4]{\frac{1}{N} \sum_{j=1}^{N}\left(P_{i, j}-A_{i}\right)^{4}}
$$

\subsection{Feature Vector and Classification}

For a color logo image, the four color moments as given in equations (8) to (11) are computed for each color channel R, $\mathrm{G}$ and $\mathrm{B}$, for modeling the color information The first and second $\mathrm{Hu}$ moments as given by equation (7) are then computed from the grayscale version of the image for modeling the shape of the logo. The final feature vector is a collection of these 14 elements and given by:

$$
F=\left\{A_{i}, S_{i}, W_{i}, K_{i}, M_{1}, M_{2}\right\}, \quad i \in\{R, G, B\}
$$

An image class is defined by the collection of a set of member images. Each class is characterized by the collection of 14element feature vectors of its member images as defined above, obtained during a training phase. The feature vector of the input test image is compared with those of the training images using Euclidean distance and is assumed to belong to that class for which the distance is minimum. For two $n$ element vectors $T=\left\{t_{1}, t_{2}, \ldots, t_{n}\right\}$ and $S=\left\{s_{1}, s_{2}, \ldots, s_{n}\right\}$ the Euclidean distance between them is given by

$$
D=\sqrt{\sum_{i=1}^{n}\left(t_{i}-s_{i}\right)^{2}}
$$

\subsection{Handling Unknown Classes}

An unknown class is one whose images do not form part of the training set. Before processing a test image for classification, the system is designed to check whether the test image belongs to a known class. To perform this check, the $\mathrm{Hu}$ moments as in equation (7) are computed for each image of the training set and represented as a 2-element vector after being scaled by appropriate amounts. Each of these vectors are compared with the corresponding vector of an input test image by computing the absolute difference between them. If the minimum of these differences is greater than a predefined threshold $T_{h}$ then the input image is considered as unknown and not submitted to the classifier.

\section{EXPERIMENTATIONS \& RESULTS 4.1 Dataset}

The dataset of color logo images used in the experimentations is collected from different websites and consists of 75 different logo images saved in BMP format, as shown below in Fig 1. 


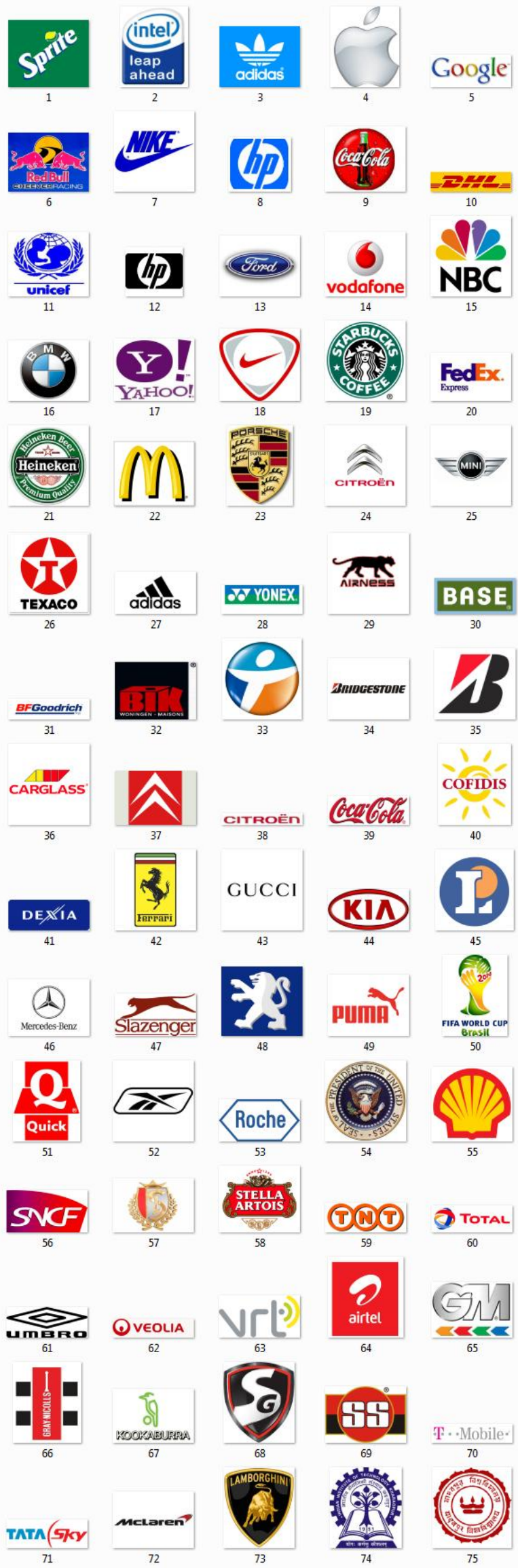

Fig 1: Sample Logo images of the dataset

\subsection{Training Phase}

The images shown in Fig. 1 are used as the training set, one image per class. The color features $A_{R}, A_{G}, A_{B}$ are computed from the color channels. Fig. 2 shows the variation of these values of the training files over all the 75 classes.


Fig 2: Variation of mean (A) for training set

The color features $S_{R}, S_{G}, S_{B}$ are computed from the color channels. Fig. 3 shows the variation of these values of the training files over all the 75 classes
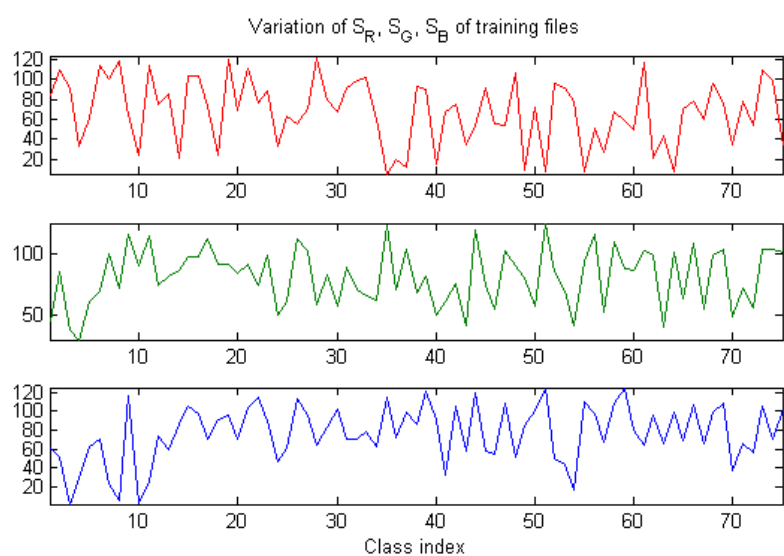

Fig 3: Variation of standard deviation (S) for training set

The color features $W_{R}, W_{G}, W_{B}$ are computed from the color channels. Fig. 4 shows the variation of these values of the training files over all the 75 classes
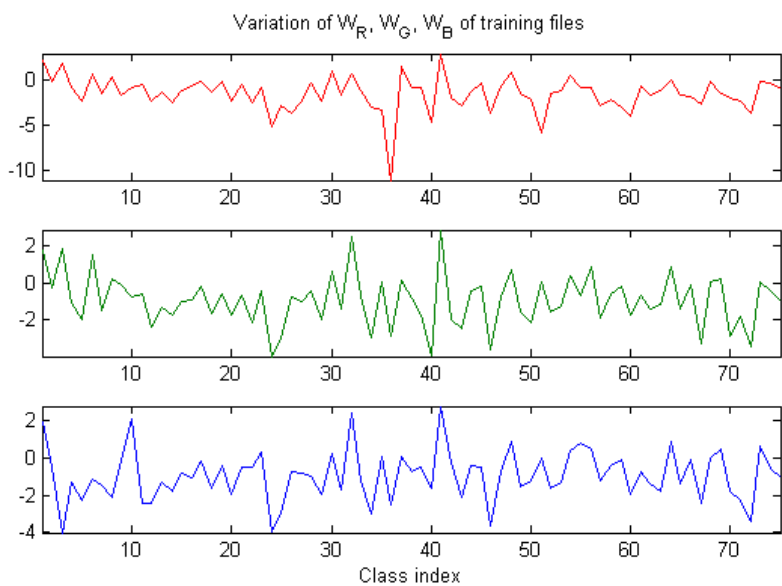

Fig 4: Variation of skewness (W) for training set 
The color features $K_{R}, K_{G}, K_{B}$ are computed from the color channels. Fig. 5 shows the variation of these values of the training files over all the 75 classes
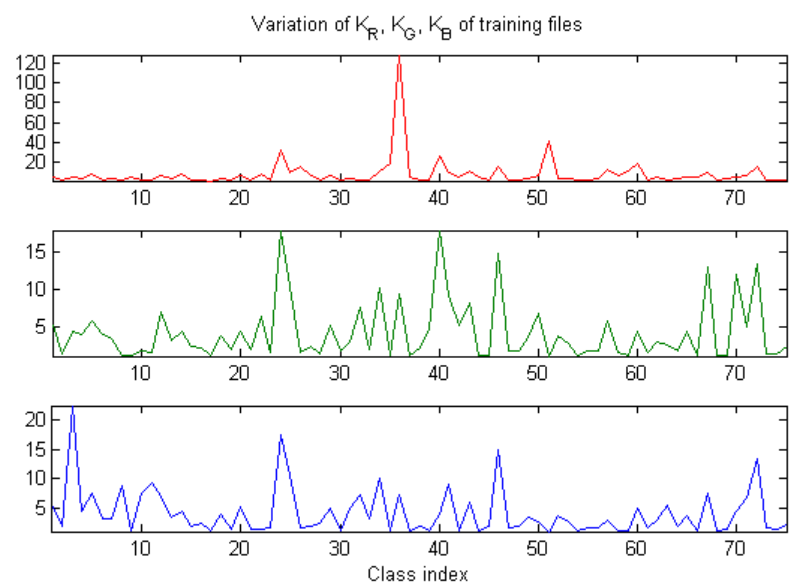

Fig 5: Variation of kurtosis (K) for training set

The shape features $M_{1}, M_{2}$ are computed from the grayscale version of the images. Fig. 6 shows the variation of these values of the training files over all the 75 classes. The values have been scaled by multiplying with scaling factors of $10^{2}$ and $10^{6}$ respectively.

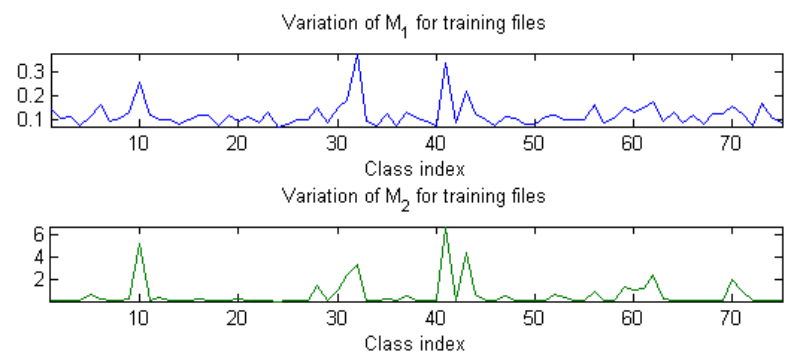

Fig 6: Variation of M1 and M2 for training set

\subsection{Testing Phase}

To introduce variations between the training and test images, 11 types of transformation are applied to each of the images viz. four rotation factors $\mathrm{R}\left(9^{\circ}, 15^{\circ}, 90^{\circ}, 180^{\circ}\right)$, five scaling factors $\mathrm{S}(0.5,0.75,1.25,1.5,2)$ and two composite transformations : rotation by $15^{\circ}$ followed by scaling of 0.75 and rotation by $90^{\circ}$ followed by scaling of 1.25 . Thus a total of $11 \times 75$ or 825 images are used as the testing set. Samples of the transformed images are shown in Fig. 6 to Fig. 8

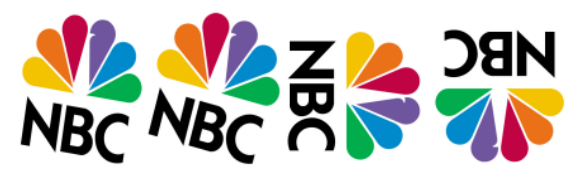

Fig 6: Sample image rotated by $9^{\circ}, 1^{\circ}, 90^{\circ}, 180^{\circ}$

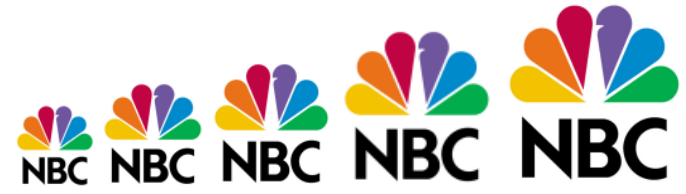

Fig 7: Sample image scaled by factors of $0.5,0.75,1.25$, $1.5,2$

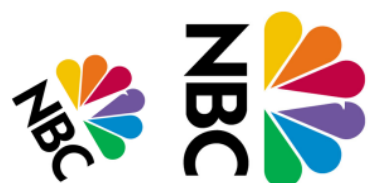

Fig 8: Sample image with composite transformations: [R(15), S(0.75)] and [R(90), S(1.25)]

To illustrate the variation of features for the transformed images, the following figure indicates the features $A_{B}, S_{B}, W_{B}, K_{B}, M_{1}, M_{2}$ for the blue channel of the test image rotated by $15^{\circ}$ and scaled by 0.75 , over all the 75 classes are shown in Fig, 9.
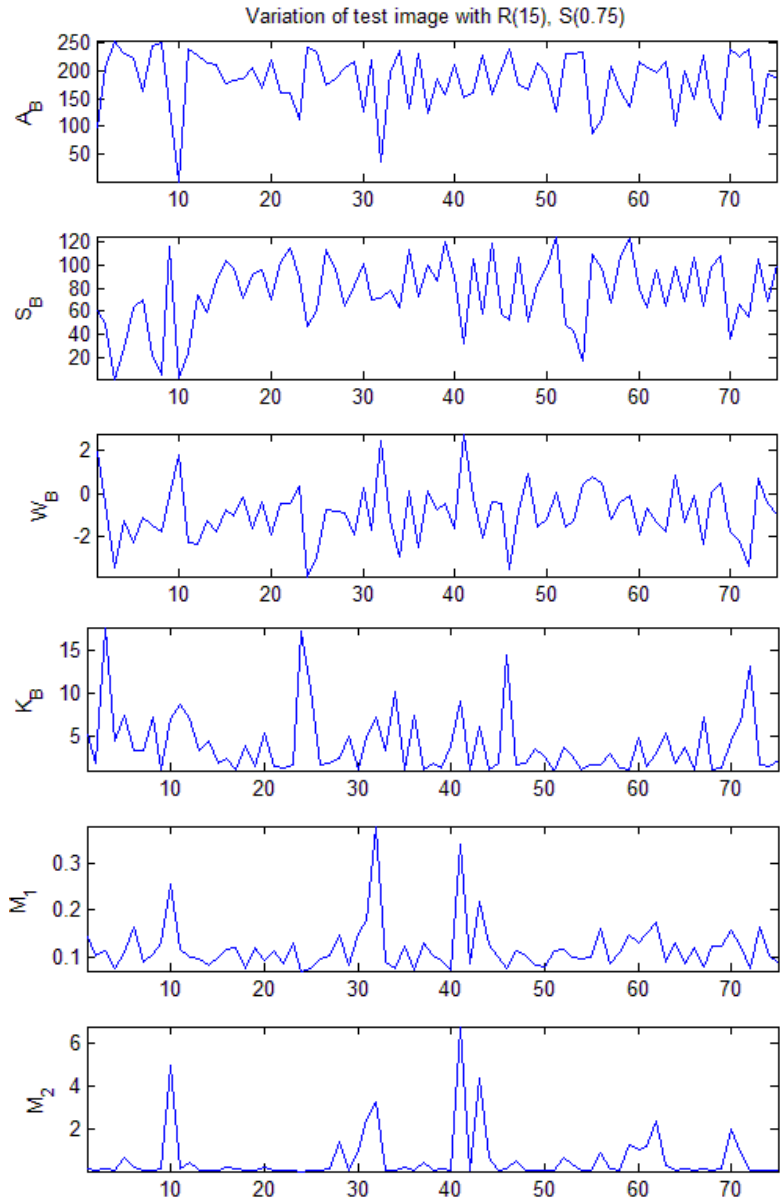

Fig 9: Variation of features for blue channel of test image with $R(15), S(0.75)$

\subsection{Classification}

Classification is performed by calculating Euclidean distance between each test vector and all the 75 training vectors. The test image is assumed to belong to the class for which distance is the minimum. Fig. 10 shows the classification plots for the test images belonging to class 25, 50, 75 having transformation R(9). Similarly, Fig. 11 shows the classification plots for the test images belonging to class 10 , 40, 70 having transformation $\mathrm{S}(0.75)$ and Fig. 12 shows the classification plots for the test images belonging to class 5, 35, 65 having composite transformation $\mathrm{R}(90) \& \mathrm{~S}(1.25)$. The minimum distance and the inferred class, is marked with a red circle in each case. 

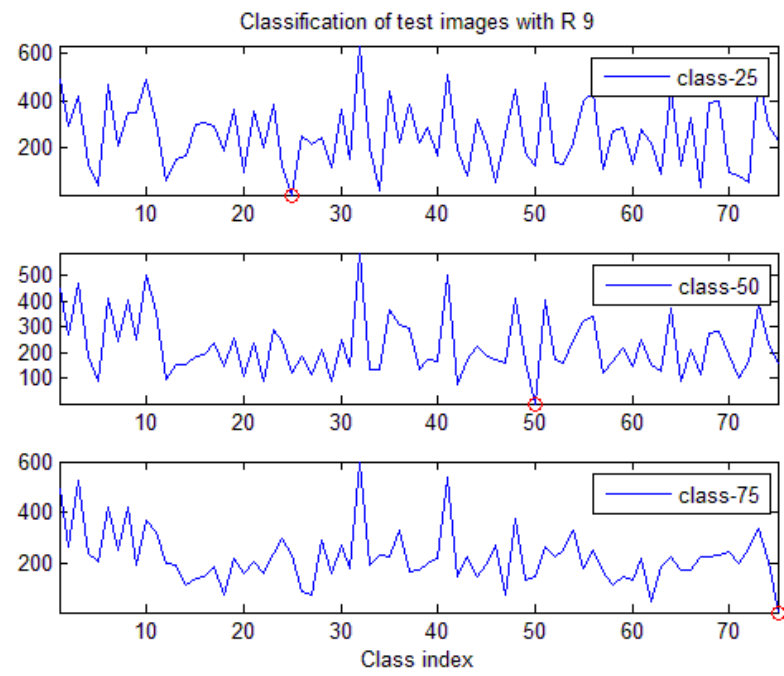

Fig. 10: Classification plots for test images belonging to classes $25,50,75$ with transformation $R(9)$.
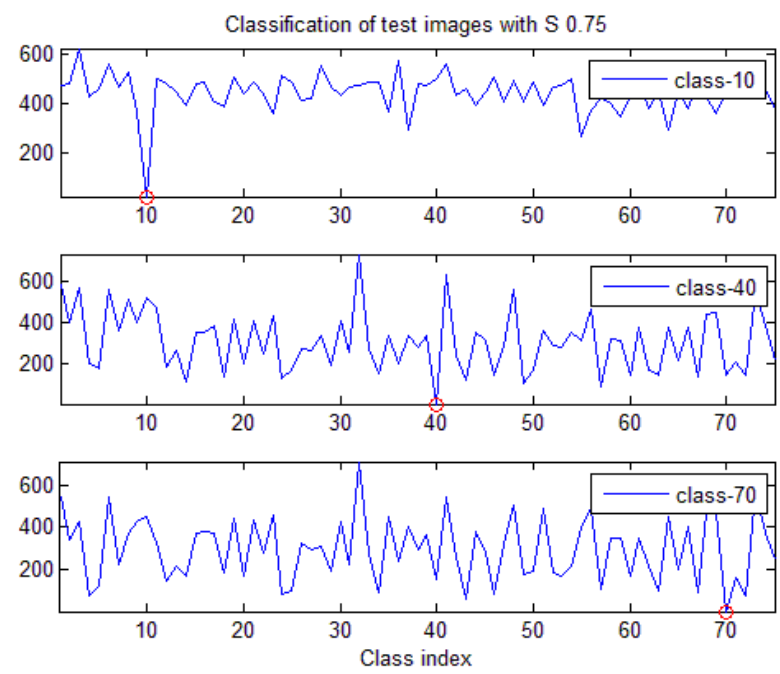

Fig. 11: Classification plots for test images belonging to classes 10, 40, 70 with transformation $S(0.75)$.
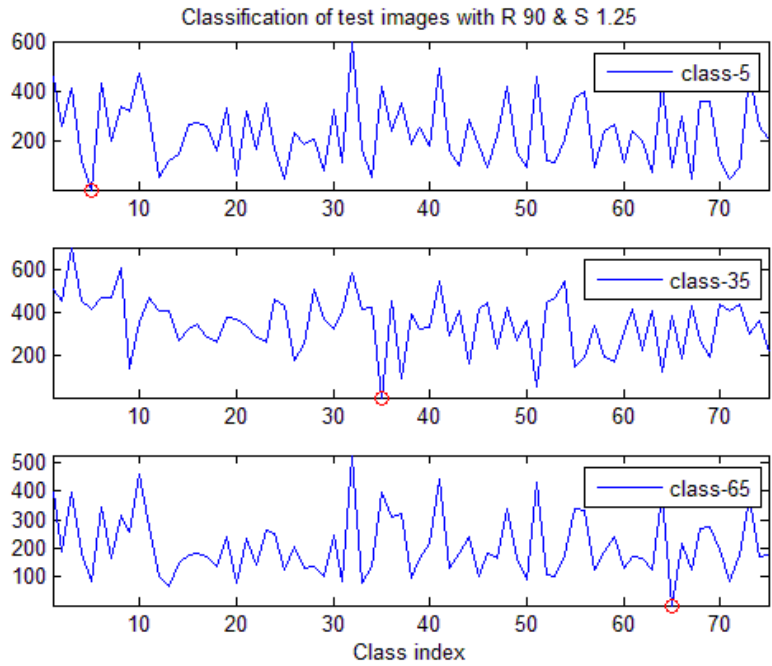

Fig. 12: Classification plots for test images belonging to classes 5, 35, 65 with transformation $R(90) \& S(1.25)$.

\subsection{Unknown Classes}

The threshold for determining unknown classes is experimentally defined as $T_{h}=0.0210$. Some of the images which have been successfully determined as unknown are shown in Fig. 13.

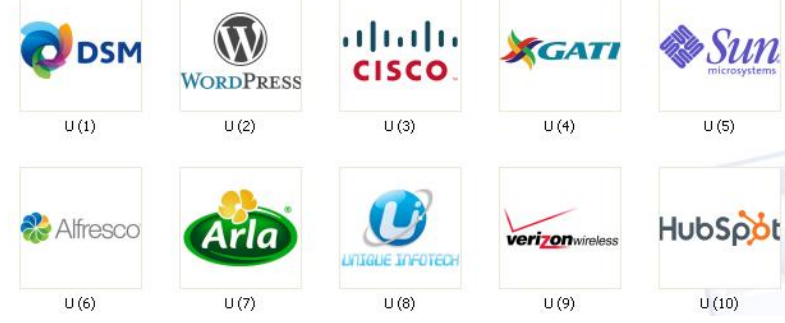

Fig. 13: Unknown logo images detected by the system

Fig. 14 depicts a plot for discriminating between 10 known and 10 unknown images by using the threshold of 0.021 .

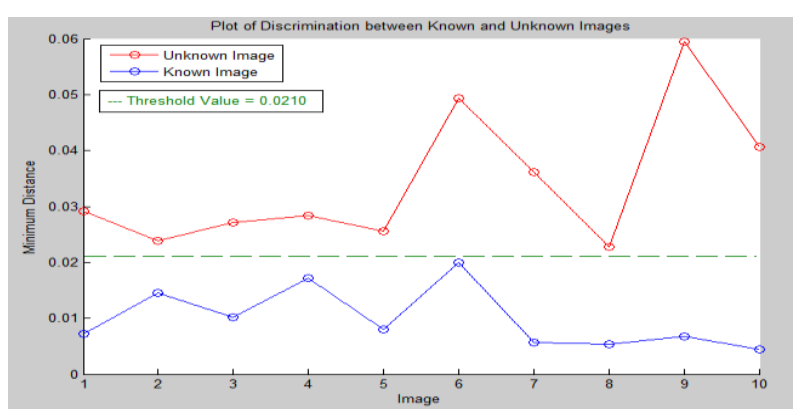

Fig. 14: Discrimination plot between known and unknown images

\subsection{Accuracy}

Recognition accuracy calculated using 75 training images and 825 testing images with combinations of various rotation and scaling factors and a number of features, is shown in Table 1.The accuracy mentioned is the overall value for all 75 classes. The last column corresponds to the 14 -element vector defined in equation (12).

Table 1: Recognition Accuracy obtained using various combination of features

\begin{tabular}{|c|c|c|c|c|c|}
\hline Features & $\begin{array}{c}\text { 12 color } \\
\text { moments }\end{array}$ & $\mathbf{M}_{\mathbf{1}}$ & $\mathbf{M}_{\mathbf{2}}$ & $\mathbf{M}_{\mathbf{1}}+\mathbf{M}_{\mathbf{2}}$ & $\begin{array}{c}\mathbf{1 2} \text { color } \\
\text { moments + } \\
\mathbf{M}_{\mathbf{1}}+\mathbf{M}_{\mathbf{2}}\end{array}$ \\
\hline \% Acc & 70.33 & 89.33 & 84 & 96 & 98.69 \\
\hline
\end{tabular}

Table 2 below shows the recognition accuracies for various transformations separately using the 14-element vector.

Table 2: Recognition Accuracy obtained for various transformations

\begin{tabular}{|l|c|}
\hline Transformations & $\begin{array}{c}\text { Recognition Accuracy } \\
\text { based on 14 element vector }\end{array}$ \\
\hline Rotation by $9^{\circ}$ & 97.33 \\
\hline Rotation by $15^{\circ}$ & 94.67 \\
\hline Rotation by $90^{\circ}$ & 100 \\
\hline Rotation by $180^{\circ}$ & 100 \\
\hline
\end{tabular}




\begin{tabular}{|l|c|}
\hline Scaling by 0.5 & 100 \\
\hline Scaling by 0.75 & 98.67 \\
\hline Scaling by 1.25 & 100 \\
\hline Scaling by 1.5 & 100 \\
\hline Scaling by 2 & 100 \\
\hline Rotation $15^{\circ}$, scaling 0.75 & 95 \\
\hline Rotation $90^{\circ}$, scaling 1.25 & 100 \\
\hline Overall & 98.69 \\
\hline
\end{tabular}

Class wise percentage accuracies are given below with the overall accuracy of $99.55 \%$ :

$100,100,100,100,100,100,100,83.33,100,100,100,100$, $100,100,100,100,91.67,100,100,100,100,100,100,100$, $100,100,100,100,100,100,91.67,100,100,100,100,100$, $100,100,100,100,100,100,100,100,100,100,100,100$, $100,100,100,100,100,100,100,100,100,100,100,100$, $100,100,100,100,100,100,100,100,100,100,100,100$, $100,100,100$

\subsection{User Interface}

For a more robust evaluation of the rotation-invariant and scale-invariant recognition characteristics of the system, a user interface designed into the system gives the user the option of rotating and scaling the image by any arbitrary amounts before submission to the recognition engine. Samples of the input and output windows are shown in Fig. 15.

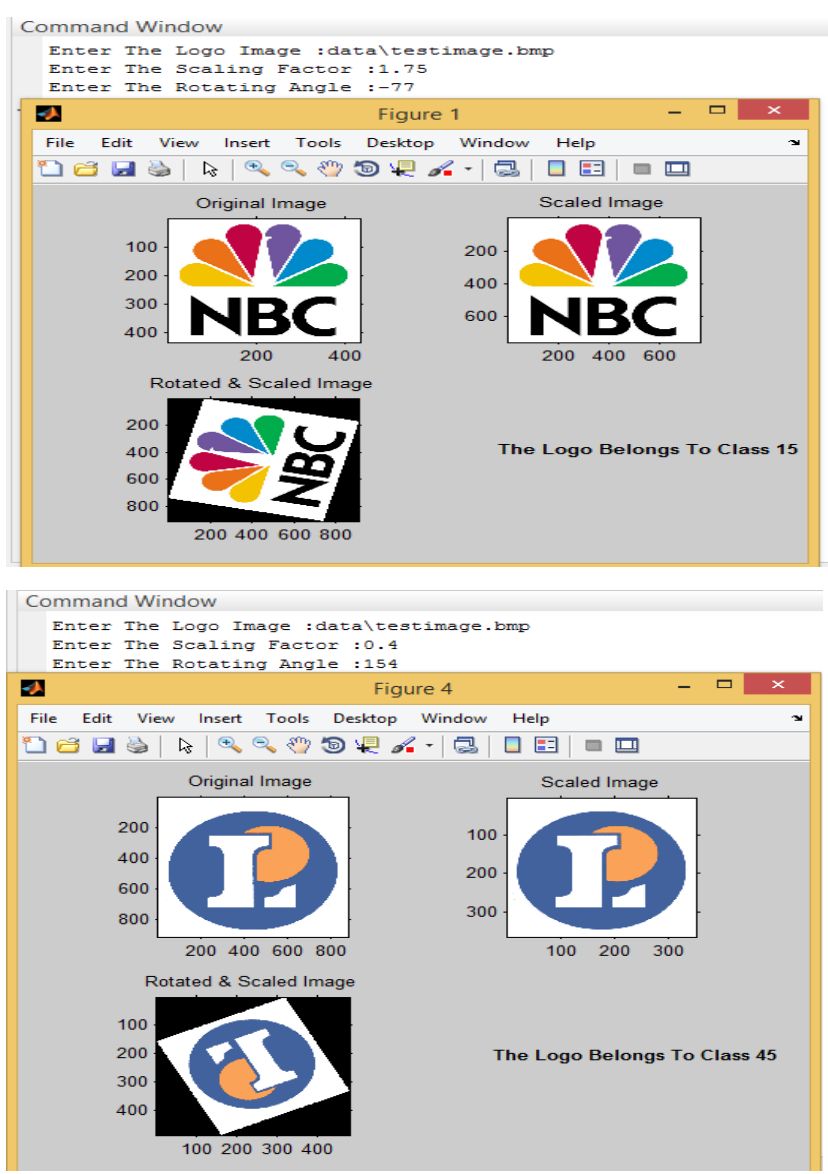

Fig. 15: Experimental Results using arbitrary variations specified by the user

\section{ANALYSIS}

Automated recognition of logo images have been done using combination of various methods. Using M1, M2 as a vector gives better result than individual M1 and M2. But the proposed approach using 14 element feature vector comprising of M1, M2 and the 12 color moments gives the best result.

To put the above results in perspective with the state of art, the following table shows comparison of the proposed approach with that of [5]. In both approaches, the transformation applied to original logos images are of 5 types, namely scaling by $0.5,0.7$ and rotation by $15^{\circ}, 30^{\circ}$, and $45^{\circ}$. After applying this transformations the results obtained for the two methods are explained in details in Table 3.

Table 3: Comparison between recognition accuracy of approaches used in [5] and proposed approach (PA):

\begin{tabular}{|l|l|l|l|l|}
\hline $\begin{array}{l}\text { Appro- } \\
\text { aches }\end{array}$ & $\begin{array}{l}\text { No. of } \\
\text { Classes }\end{array}$ & $\begin{array}{l}\text { No. of } \\
\text { images }\end{array}$ & $\begin{array}{l}\text { Process- } \\
\text { ing } \\
\text { Time }\end{array}$ & $\begin{array}{l}\text { Combined } \\
\text { Recognition } \\
\text { Accuracy \% } \\
\text { (Using all 5 } \\
\text { transformati } \\
\text { ons) }\end{array}$ \\
\hline $\begin{array}{l}\text { FMT } \\
\text { Approach } \\
\text { in [5] }\end{array}$ & 46 & 230 & $3.92 \mathrm{~min}$ & 84.84 \\
\hline $\begin{array}{l}\text { LPT } \\
\text { Approach } \\
\text { in [5] }\end{array}$ & 46 & 230 & 9.73 min & 86.83 \\
\hline $\begin{array}{l}\text { Moment } \\
\text { Invariant } \\
\text { Approach } \\
\text { in [5] }\end{array}$ & 46 & 230 & 9.30 min & 90.27 \\
\hline PA & $\mathbf{7 5}$ & $\mathbf{3 7 5}$ & $\mathbf{2 . 4 5}$ min & $\mathbf{9 8 . 9 3}$ \\
\hline
\end{tabular}

From the above table it can be inferred that the proposed approach outperforms all the methods mentioned in approach [5] both in terms of recognition accuracy and processing time and also in terms of database size. Also another constraint of [5] is that their images needs to be resized to $256 \times 256$ before extracting features.

Further Log Polar Transform (LPT) and Fourier Mellin Transform (FMT) suffer from loss of information due to conversion into log polar form, as Cartesian coordinates cannot be mapped one-to-one into the log-polar coordinate space. Therefore average of the surrounding pixels is used in mapping in log polar space, which results in loss of information. Also LPT and FMT have low recognition rates as they are affected by interpolation artifacts while rotating the images. The proposed approach solves all the above problems showing good results for both rotation and scaling.

In [7] Angular Radial Transform (ART) is used to classify logos using transformations with rotation of $30^{\circ}, 60^{\circ}, 90^{\circ}$ and $180^{\circ}$ and scaling factors of $0.5,0.75$ separately. Following table 4 and 5 show in details the comparison between two methods.

Table 4: Comparison between recognition accuracy of approaches under different rotation angles

\begin{tabular}{|l|l|l|}
\hline Rotation Angle & ART System[7] & PA \\
\hline $30^{\circ}$ & 73.33 & $\mathbf{1 0 0}$ \\
\hline $60^{\circ}$ & 61.90 & $\mathbf{1 0 0}$ \\
\hline
\end{tabular}




\begin{tabular}{|l|l|l|}
\hline $90^{\circ}$ & 66.67 & $\mathbf{1 0 0}$ \\
\hline $180^{\circ}$ & 92.38 & $\mathbf{1 0 0}$ \\
\hline Average & 73.57 & $\mathbf{1 0 0}$ \\
\hline
\end{tabular}

Table 5: Comparison between recognition accuracy of approaches under different scaling factors

\begin{tabular}{|l|l|l|}
\hline Scaling Factor & ART System[7] & PA \\
\hline 0.5 & 53.33 & $\mathbf{9 8 . 6 7}$ \\
\hline 0.75 & 78.14 & $\mathbf{1 0 0}$ \\
\hline Average & 65.74 & $\mathbf{9 9 . 3 3}$ \\
\hline
\end{tabular}

The major drawback of ART is that for segmented planar objects from real images, one have to take into account unspecified rotations. As the basis functions are symmetrical in the angular direction, the invariance is inherent for planar rotations. Unspecified rotations induce a real deformation of the original shape due to the perspective projection into the image plan. More precisely ART is not invariant to all rotations, but in case of proposed approach, user can apply any rotations of his choice and still correctly recognize the logo.

In [8] various methods, like Zernike Moments (ZM), Legendre Moments (LM), Radial Tchebichef Moments (RTM) have been used for logo recognition using rotation and scaling transformations. Various rotation transformations such as $30^{\circ}, 60^{\circ}, 90^{\circ}$ and composite transformations such as scaling \& rotation are performed. The comparison of the above method and proposed approach is shown in the table 6 and 7.

Table 6: Comparison between recognition accuracy of approaches under different rotation angles

\begin{tabular}{|l|l|l|l|l|}
\hline $\begin{array}{l}\text { Rotation } \\
\text { Angle }\end{array}$ & $\begin{array}{l}\text { LM } \\
\text { approach in } \\
{[\mathbf{8}]}\end{array}$ & $\begin{array}{l}\text { ZM } \\
\text { approach } \\
\text { in [8] }\end{array}$ & $\begin{array}{l}\text { RTM } \\
\text { approach } \\
\text { in [8] }\end{array}$ & PA \\
\hline $30^{\circ}$ & 30 & 20 & 90 & $\mathbf{9 9}$ \\
\hline $60^{\circ}$ & 28 & 20 & 72 & $\mathbf{9 5}$ \\
\hline $90^{\circ}$ & 6 & 98 & 100 & $\mathbf{1 0 0}$ \\
\hline $\begin{array}{l}\text { Average } \\
\text { Rate }\end{array}$ & 21.33 & 46 & 87.33 & $\mathbf{9 8}$ \\
\hline
\end{tabular}

Table 7: Comparison between recognition accuracy of approaches under different rotation and scaling

\begin{tabular}{|l|l|l|l|l|}
\hline Variations & $\begin{array}{l}\text { LM } \\
\text { approach } \\
\text { in [8] }\end{array}$ & $\begin{array}{l}\text { RTM } \\
\text { approach } \\
\text { in [8] }\end{array}$ & $\begin{array}{l}\text { ZM } \\
\text { approach } \\
\text { in [8] }\end{array}$ & PA \\
\hline $\begin{array}{l}\text { Rotation } 60^{\circ}, \\
\text { Scaling } 0.75\end{array}$ & 28 & 36 & 20 & $\mathbf{1 0 0}$ \\
\hline $\begin{array}{l}\text { Rotation } 90^{\circ}, \\
\text { Scaling } 1.25\end{array}$ & 6 & 52 & 98 & $\mathbf{1 0 0}$ \\
\hline $\begin{array}{l}\text { Average } \\
\text { Rate }\end{array}$ & 17 & 49 & 59 & $\mathbf{1 0 0}$ \\
\hline
\end{tabular}

The main disadvantages of Zernike moments are that the image coordinate space must be transformed to the domain where the orthogonal polynomial is defined. The continuous integrals in Zernike moments must be approximated by discrete summations. This approximation not only leads to numerical errors in the computed moments, but also severely affects the analytical properties such as rotational invariance. Computational complexity of the radial Zernike polynomial increases as the order becomes large.
Comparing the results in the above 3 methods it can be clearly stated the proposed approach clearly outperforms the other approaches in case of rotation and scaling thus proving the robustness of the proposed system.

Table 8: Comparison Of Various Approaches with rotation and scaling transformations

\begin{tabular}{|l|c|c|c|c|c|l|l|}
\hline & RTM & ZM & LM & ART & FMT & LPT & PA \\
\hline $\begin{array}{l}\text { \% } \\
\text { Acc }\end{array}$ & 53.75 & 59.7 & 63 & 76.6 & 84.8 & 86.8 & $\mathbf{9 8 . 6 9}$ \\
\hline
\end{tabular}

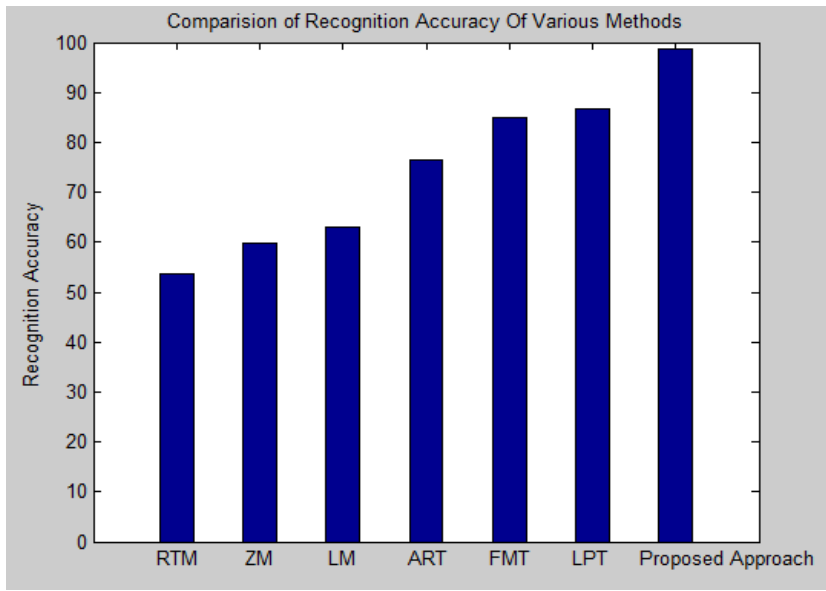

Fig. 16: Comparison Plot of Recognition Accuracy of Various Methods

\section{CONCLUSIONS AND FUTURE SCOPES}

This paper proposes an automated system for rotation and scale invariant color logo recognition. It uses 1st and 2nd Order Invariant Moments and 4 color moments namely mean, standard deviation, skewness, and kurtosis for recognizing color logo images. The accuracy of the proposed approach is comparable to those reported in contemporary works. Salient features of the proposed technique include a low dimensional feature representation, low computational overheads involving only scalar arithmetic and high recognition accuracies. This enables recognition and identification possible in real-time applications. Another important characteristic of this work is the robustness, scalability and flexibility of the method which provides satisfactory results even after rotating and scaling the image thus distorting it. The method has been used with uniform scaling, but in future non uniform scaling i.e. scaling differently in both directions may be introduced to change the total orientation of the image and then recognizing it to increase the robustness of the algorithm.

Future work would involve research in the following directions: (1) applying non-uniform scaling and other transformations to distort the image and then recognize it, (2) introducing various color variations such as changing color tint or other color properties and then recognizing the images, (3) using various texture features other than shape and color features to recognize distorted images with a good recognition accuracy, and (4) using other dataset or other logo images to increase the scalability of the proposed approach. 


\section{REFERENCES}

[1] Soffer, A. and Samet, H. 1998. Using negative shape features for logo similarity matching. In Proceedings of IEEE Fourteenth International Conference on Pattern Recognition, pp. 571-573.

[2] Mikolajczyk, K. and Schimd, C. 2005. A Performance Evaluation of Local Descriptors. In Proceedings of the IEEE International Conference on Pattern Analysis and Machine Intelligence, pp. 1615-1630.

[3] Xia, L., Qi, F. and Zhou, Q. 2008. A Learning-based Logo Recognition Algorithm Using SIFT and Efficient Correspondence Matching. In Proceedings of the IEEE International Conference on Information and Automation (ICIA), pp. 1767-1772.

[4] Li, H. and Zheng, Y. 2009. A Research on Logo Recognition in the E-business. In Proceedings of the IEEE International Conference on International Computation \& Technology (ICICTA), pp. 281-285.
[5] Arafat, S. and Saleem, M. 2009. Comparative Analysis of Invariant Schemes for Logo Classification. In Proceedings of the IEEE International Conference on Emerging Technologies (ICET), pp. 256-261.

[6] Zhu, G. and Doermann, D. 2009. Logo Matching for Document Image Retrieval. In Proceedings ofTenth International Conference on Document Analysis and Recognition, pp. 606-610.

[7] Wahdan, O., Omar, K. and Nasrudin, M. 2011. Logo Recognition System Using Angular Radial Transform Descriptors.Journal of Computer Science, pp. 14161422.

[8] Zhang, Z., Wang, X., Anwar, W. and Jiang, Z. 2014. A Comparison of Moments-Based Logo Recognition Methods. Hindawi Publishing Corporation.

[9] $\mathrm{Hu}, \mathrm{M}-\mathrm{K}$. 1962. Visual pattern recognition by moment invariants. In Proceedings of IRE Transactions on Information Theory, pp. 179-187. 